\title{
DETERMINACIÓN DEL PAPEL DE LAS CARACTERÍSTICAS DEMOGRÁFICAS DE LOS CONSUMIDORES EN EL PROCESO DE FORMACIÓN DE LEALTAD BASADA EN ASPECTOS SOCIALMENTE RESPONSABLES
}

\author{
Patricia Martínez \\ Ignacio Rodríguez del Bosque
}

Universidad de Cantabria

\section{RESUMEN}

La responsabilidad social corporativa (RSC) se ha convertido en un elemento crucial en la estrategia corporativa de las compañías hoteleras. Una de las principales razones es que el desarrollo de este tipo de iniciativas favorece el desarrollo de mayores niveles de lealtad entre los consumidores. Igualmente, las características demográficas de los consumidores son una línea de investigación que tradicionalmente ha centrado la atención de multitud de investigadores por considerarse herramientas útiles para la segmentación de los mercados y la caracterización de los consumidores. Sin embargo, son pocos los estudios que han tratado de explorar el papel que juegan las características demográficas de los consumidores en sus respuestas comportamentales basadas en aspectos de RSC. De este modo, el objetivo de esta investigación es analizar el papel de tres características demográficas -género, edad y nivel educativo- en un modelo conceptual que relaciona las percepciones de RSC y la lealtad de los consumidores. Los resultados revelan que estas variables demográficas moderan diversas relaciones del modelo propuesto.

Palabras clave: Responsabilidad social corporativa; lealtad del consumidor; características demográficas.

Fecha de recepción: 2 de enero de 2017.

Fecha de aceptación: 11 de octubre de 2017.

Departamento de Administración de Empresas. Universidad de Cantabria. Avenida de los Castros, s/n. 39005 SANTANDER (España).E-mail: martinezrp@unican.es, rbosquei@unican.es 


\title{
Determination of the role of consumers' demographic characteristics in the loyalty formation process based on socially responsible aspects
}

\begin{abstract}
Corporate social responsibility (CSR) has become a crucial element in the corporate strategy of hotel companies. One of the main reasons is that the development of such initiatives favors the development of higher levels of customer loyalty. Similarly, customers' demographic characteristics are a line of research that has traditionally focused the attention of academics since these features are considered useful tools for market segmentation and customers' characterization. However, few studies have attempted to explore the role of demographic characteristics of consumers in their behavioral responses based on CSR aspects. Thus, the objective of this study is to analyze the role of three demographic characteristics -gender, age and educational level- in a conceptual model that relates customers' perception of CSR and consumer loyalty. The results reveal that these demographic variables moderate various relationships in the proposed model.
\end{abstract}

Keywords: Corporate social responsibility; customer loyalty; demographic characteristics.

\section{INTRODUCCIÓN}

El sector hotelero es una de las industrias con mayor crecimiento internacional y uno de los pilares de la industria turística mundial. El sector hotelero es una fuente de beneficios no sólo en las esferas económica y social sino también en los dominios medioambiental y cultural debido a su gran influencia en distintos ámbitos como la creación de empleo, mejora de infraestructuras y la mejora de la compresión entre distintas culturas (Bohdanowicz y Zientara, 2009). Sin embargo, esta industria también ha recibido multitud de críticas por su crecimiento desorganizado y por ser el origen de una serie de situaciones no deseables como la contaminación, la pérdida de biodiversidad o el incumplimiento de las normas laborales, entre otros (Chan, 2011). Por ello, la responsabilidad social corporativa (RSC) se configura como una estrategia adecuada para que las empresas del sector minimicen estos impactos negativos al tiempo que incrementan sus efectos positivos tanto en el medioambiente como en las comunidades locales en las que operan (Martínez et al., 2012). Así, el concepto de RSC se define como la asunción por parte de las compañías de una serie de responsabilidades que van más allá de los accionistas y los clientes, teniendo en cuenta a la sociedad en su conjunto (Holloway, 2004).

Por ello, hoy en día multitud de empresas del sector hotelero han puesto en marcha diversas iniciativas para mostrar su compromiso con esta filosofía corporativa. Una de las principales razones argumentadas para el gran interés por los aspectos socialmente responsables es el potencial efecto positivo en el comportamiento de los consumidores, en concreto en su nivel de lealtad (Martínez y Rodríguez del Bosque, 2013) sobre todo en un momento en que las empresas hoteleras se enfrentan a un entorno extremadamente 
competitivo y con crecientes expectativas por parte de los consumidores. En la actualidad multitud de compañías hoteleras están teniendo dificultades para incrementar su cuota de mercado debido a la creciente competencia internacional y al exceso de oferta en los mercados maduros (So et al., 2013). Por ello, las compañías del sector están desarrollando estrategias centradas en la retención de sus clientes.

No obstante, y al objeto de atraer y retener a los consumidores "más responsables" es necesario identificar los factores que les definen. Recientes investigaciones siguieren que no solo el altruismo incide a la hora de comprar productos "sostenibles" o "responsables" sino que el consumo de este tipo de productos y el apoyo (a través de la compra y la recomendación) a las empresas que apuestan por la RSC viene determinada por factores demográficos y socio-económicos, así como culturales (Balderjahn, 1998; Pivato et al., 2008). En este sentido, las características socio-demográficas género, edad y nivel educativo han sido identificadas como las variables más importantes que influyen en la relación de los consumidores con las empresas y en la lealtad de los consumidores hacia sus productos o servicios (Homburg y Giering, 2001; Walsh et al., 2008). Sin embargo, ningún estudio hasta la fecha ha analizado el papel que juegan estas variables en el proceso de formación de lealtad basada en aspectos socialmente responsables. Por lo tanto, el objetivo fundamental de este estudio es evaluar el impacto de estas tres características socio-demográficas en la lealtad de los consumidores de establecimientos hoteleros basados en aspectos de RSC.

\section{REVISIÓN DE LITERATURA}

Para cumplir los objetivos de este estudio, los autores se basan en el modelo desarrollado por Martínez y Rodríguez del Bosque (2013) para comprender como los consumidores forman su grado de lealtad a través de aspectos socialmente responsables. Estos autores ofrecen un modelo causal que explica el efecto de la RSC en el proceso de formación de lealtad de los consumidores incluyendo (1) la confianza del consumidor, (2) su nivel de identificación con la empresa y (3) su grado de satisfacción como variables mediadoras. Los resultados de este estudio confirman el valor estratégico que supone para las empresas del sector hotelero la participación en actividades de RSC puesto que los consumidores (1) perciben que las empresas que desarrollan iniciativas socialmente responsables operan de una manera más honesta, (2) están más dispuestos a relacionarse con estas compañías como un modo de incrementar su autoestima y mejora personal y (3) desarrollan mayores niveles de satisfacción.

A pesar de que este modelo ha sido analizado en diversos contextos, diversos autores concluyen que el efecto de posibles efectos moderadores en el proceso de formación de la lealtad de los consumidores ha sido ignorado en la literatura académica (Evanschitzky y Wunderlich, 2006; Walsh et al., 2008). Por ello, el presente estudio propone examinar el efecto del género, edad y nivel educativo de los consumidores a la hora de desarrollar su lealtad a las compañías que desarrollan iniciativas de RSC. En este sentido, las características demográficas de los consumidores han sido consideradas tradicionalmente como un elemento fundamental para comprender el comportamiento de los consumidores debido principalmente a su efecto en el 
proceso de selección, la evaluación de productos y servicios, y en el nivel de lealtad (Vasquez-Carrasco y Foxal, 2006). Siguiendo estudios previos, si una variable $X$ (p.e., percepción de comportamiento responsable) está relacionada con otra variable $Y$ (p.e., lealtad de los consumidores), pero sólo bajo ciertas condiciones de la variable $Z$ (p.e., edad), entonces $Z$ es una variable moderadora (Baron y Kenny, 1986).

\subsection{Efecto de las variables demográficas en el proceso de formación de lealtad de los consumidores basados en aspectos de RSC}

La mayoría de los estudios sobre las diferencias de género en el comportamiento social se basan en la Teoría de los Roles Sociales y en la psicología evolutiva. Estas teorías se consideraran como los principales marcos para explorar las diferencias existentes en los patrones y comportamientos de los individuos debido a su género. Estas teorías sugieren que hombres y mujeres socializan de una manera diferente y desempeñan papeles diferentes en nuestra sociedad (Archer, 1996; Buss, 1996). Estudios previos indican que el género desempeña un papel fundamental en el mantenimiento de patrones en los comportamientos sociales (Han et al., 2009; Luiz y de Matos, 2015; Saad y Gill, 2000).

El impacto del género en el comportamiento de compra se encuentra ampliamente reconocido en la literatura de marketing y del comportamiento del consumidor. En particular, varios estudios han explorado el papel de las diferencias de género en el consumo de diversos productos y servicios (Homburg y Giering, 2001; Laroche et al., 2001; Mittal y Kamakura, 2001; Walsh et al., 2008). De acuerdo con Eagly (1987), las mujeres están más preocupadas por el bienestar de otros que los hombres. A este respecto, estudios previos sugieren que las mujeres que participan en las decisiones de compra son más propensas a prestar mayor intención atención a las interacciones personales y las relaciones interpersonales (Konrad et al., 2000). De acuerdo con estas ideas, Laroche et al. (2001) comprobaron empíricamente que las mujeres estaban más preocupadas por el medio ambiente que los hombres. Del mismo modo, Han et al. (2009) en su estudio sobre el proceso de toma de decisiones basado en aspectos medioambientales descubrieron que la relación entre las actitudes hacia este tipo de iniciativas y el desarrollo de un comportamiento "ecológico" (en términos de intención de compra, recomendación y disposición a pagar un precio mayor para promover estas iniciativas) era mayor para las mujeres y los consumidores con una edad elevada. Estos resultados pueden explicarse por la Teoría del Rol Social argumentando que las mujeres son más responsables y obedientes mientras que los hombres tienden a ser más autosuficientes y agresivos (Saad y Gill, 2000).

Sobre la base de estas ideas, los autores proponen su primera hipótesis de investigación en relación con el papel moderador del género de los consumidores en el proceso de formación de lealtad basada en aspectos de RSC. No obstante, los autores proponen todas las hipótesis, de tal manera que las diferencias en la forma en que los consumidores desarrollan su lealtad se identifican directamente a través del análisis empírico, no proponiéndose teóricamente. Esto es debido a que ningún estudio hasta la fecha ha explorado el papel moderador de las características demográficas de los consumidores 
en su proceso de formación de lealtad basado en aspectos socialmente responsables. En consecuencia, la primera hipótesis de investigación dice así:

H1: El género de los consumidores moderará su proceso de formación de lealtad basada en aspectos socialmente responsables.

La edad es otro factor determinante de los comportamientos de compra. No obstante, los resultados de estudios previos sobre la influencia de la edad en el comportamiento de los consumidores y en su proceso de toma de decisiones son variados y no concluyentes. Por un lado, diversas investigaciones sugieren que las personas de mayor edad son más conservadores, mientras que los consumidores más jóvenes tienden a ser más innovadores y aceptan en mejor medida nuevos productos y servicios. Por otra parte, estudios basados en la Teoría del Procesamiento de la Información indican que los individuos más jóvenes tienen a buscar información nueva y alternativa (Evanschitzky y Wunderlich, 2006; Gilly y Zeithaml, 1985), mientras que las personas mayores son más propensas a confiar en la información existente sobre las organizaciones y su oferta, sobre todo debido a que las capacidades de procesamiento de información disminuyen con la edad (Evanschitzky y Wunderlich, 2006; Gilly y Zeithaml, 1985). El importante papel de la edad en el proceso de compra basado en cuestiones responsables también se identifica en estudios previos (Han et al., 2009; Pérez y Rodríguez del Bosque, 2013; Roberts, 1996; Sandhal y Robertson, 1989; Wakefield y Baker, 1998). En general, estos estudios demuestran que los consumidores que con mayor frecuencia apoyan las iniciativas de RSC tienen una edad mayor que la media (Pivato et al., 2008; Sandhal y Robertson, 1989). Dada esta evidencia empírica, podemos esperar que la edad de los consumidores posea un papel relevante en la formación de su lealtad basada en aspectos de RSC. En concreto, se propone que:

H2: Le edad de los consumidores moderará su proceso de formación de lealtad basada en aspectos socialmente responsables.

Por último, los autores analizan la influencia del nivel educativo de los consumidores en el su proceso de formación de lealtad basada en la RSC. Diversas investigaciones han demostrado que la lealtad de los consumidores se relaciona de manera inversa con su nivel de educación, a mayor nivel educativo de los consumidores menor nivel de lealtad (Mittal y Kamakura, 2001). En general, los consumidores con mayores niveles educativos desarrollan procesos cognitivos más detallados y llevan a cabo búsquedas de información más extensas. En este sentido, las personas con educación superior se espera que sean más conscientes de los productos de la competencia y que, en general, sean capaces de evaluar más opciones.

De un modo similar, estudios previos han identificado al consumidor con una mayor orientación social como una persona altamente educada. En general, se demuestra que los consumidores socialmente responsables están mejor educados que la población general (Diamantopoulos et al., 2003; Pivato et al., 2008; Roberts, 1996). Por ejemplo, Quazi (2003) observó que las personas con un grado educativo alto tendían a desa- 
rrollar percepciones de RSC más elaboradas. Igualmente, investigaciones previas han demostrado que la educación no sólo determina la orientación en cuanto a iniciativas socialmente responsables (Kelley et al., 1990), sino también las actitudes hacia este tipo de iniciativas (Pérez y Rodríguez del Bosque, 2013). De este modo, proponemos la tercera hipótesis de investigación:

H3: El nivel educativo de los consumidores moderará su proceso de formación de lealtad basada en aspectos socialmente responsables.

\section{METODOLOGÍA}

\subsection{Muestra}

Para diseñar la muestra de la investigación se recurrió a un muestreo no probabilístico, en concreto a un muestreo por conveniencia, ya que no se pudo tener acceso a un censo de los clientes de establecimientos hoteleros mayores de 18 años de la Comunidad Autónoma de Cantabria (España). No obstante, con el objeto de garantizar una mayor representatividad de los datos se utilizó un muestro por cuotas basado en el género y la edad de los encuestados, que recoge el Instituto Nacional de Estadística en el Padrón Municipal (2010). De este modo, los porcentajes de la muestra replican el perfil de la población. Como método de recogida de información se diseño un cuestionario estructurado. El trabajo de campo se realizó en el año 2011 con la colaboración de estudiantes universitarios cursando el Grado en Administración y Dirección de Empresas de la Universidad de Cantabria. En este sentido, cada estudiante tuvo que realizar encuestas a varias personas siguiendo patrones específicos de género y edad, pudiendo elegir entre parientes, amigos o conocidos como encuestados siempre y cuando cumplieran con estos criterios. Así mismo explicaron el propósito del estudio y preguntaron si los encuestados estaban dispuestos a participar en la encuesta administrada personalmente. Para concluir, los encuestados tuvieron que responder a una pregunta "filtro" para verificar que habían sido clientes de un establecimiento hotelero en el último año. Tras eliminar cuestionarios incompletos y erróneos se obtuvieron un total de 382 cuestionarios válidos.

La Tabla 1 muestra el perfil de la muestra, que como ya hemos comentado se segmentó atendiendo a las categorías de dos de las variables moderadoras escogidas. Así, los consumidores se agruparon por género (hombres vs. mujeres), edad (18-45 vs. >45) y nivel educativo (estudios universitarios vs. no estudios universitarios). Siguiendo las ideas de Pérez y Rodríguez del Bosque (2015), los autores escogieron la edad de 45 como edad de corte para los análisis ya que estudios previos han confirmado que esta edad permite a los investigadores identificar las mayores diferencias en el nivel de conocimiento sobre aspectos socialmente de entre los consumidores. En cuanto al nivel educativo, los autores decidieron diferenciar entre consumidores con estudios y sin estudios universitarios ya que es una de las clasificaciones más comunes en estudios que han evaluado el conocimiento y las actitudes hacia las percepciones de RSC (Pérez y Rodríguez del Bosque, 2015). Para concluir, el cuestionario final fue evaluado por dos profesores familiarizados con las propuestas de investigación de este estudio para asegurar la validez de contenido. 


\section{Tabla 1}

PERFIL DE LA MUESTRA

\begin{tabular}{llllll}
\hline & $\mathrm{N}$ & $\%$ & & $\mathrm{~N}$ & $\%$ \\
\hline Género: & & & Edad: & & \\
Masculino & 190 & 49.8 & $18-24$ & 38 & 10 \\
Femenino & 192 & 50.2 & $25-34$ & 76 & 19.9 \\
& & & $35-44$ & 72 & 18.8 \\
& & & $45-54$ & 72 & 18.9 \\
& & & $55-64$ & 53 & 13.8 \\
& & & 65 & 71 & 18.6 \\
\hline Ocupación: & & & Nivel educativo: & & \\
Estudiante & 54 & 14.1 & Sin educación & 31 & 8.1 \\
Trabajador (por cuenta ajena) & 52 & 13.7 & Básica & 87 & 22.9 \\
Trabajador ( por cuenta ajena) & 151 & 39.4 & FP/COU/BUP & 114 & 29.8 \\
Jubilado/pensionista & 67 & 17.5 & Universitarios medios & 78 & 20.3 \\
Desempleado & 21 & 5.6 & Universitarios superiores & 72 & 18.9 \\
Ama de casa & 37 & 9.7 & & & \\
\hline
\end{tabular}

\subsection{Medidas}

Para la medición de las variables utilizadas en el modelo conceptual se utilizaron escalas previamente testadas en la literatura académica utilizando escalas Likert de 7 puntos $(1=$ totalmente en desacuerdo y $7=$ totalmente de acuerdo). Las asociaciones de RSC se midieron a través de la escala propuesta por Brown y Dacin (1997). El nivel de identificación del consumidor con la empresa se midió a través de la escala desarrollada por Mael y Ashforth (1992) mientras que para la satisfacción de consumidor se utilizó la escala adaptada de Cronin et al. (2000). Para la confianza del consumidor se utilizaron las escalas desarrolladas por Morgan y Hunt (1994) y Sirdeshmuhk et al. (2002). Finalmente, para la medición de la lealtad de los consumidores se utilizaron varias escalas adaptadas (Sirdeshmuhk et al., 2002; Zeithaml et al., 1996). Las escalas utilizadas pueden consultarse en el Apéndice.

Para concluir, el cuestionario final utilizado fue evaluado para asegurar la validez de contenido de las medidas. Así, dos académicos expertos en el área de turismo sostenible y dieciséis estudiantes especializados en el sector turístico ofrecieron comentarios sobre la comprensión de las escalas, su redacción y ambigüedad y cualquier otra debilidad del cuestionario.

\section{RESULTADOS}

Al objeto de testar las relaciones entre los constructos se empleó la metodología de ecuaciones estructurales (SEM - Structural Equation Modelling). Una de las ventajas de esta metodología es que permite testar relaciones entre variables latentes que se evalúan a partir de múltiples medidas. En este estudio, la secuencia seguida para el análisis de los datos corresponde a los dos pasos propuestos en la literatura: (1) el modelo de medida y (2) el modelo estructural (Anderson y Gerbing, 1988). Así, el modelo de medida trata de analizar si los conceptos teóricos están medidos correctamente a través de las variables observadas. 
Este análisis se realiza a través de los atributos de validez y fiabilidad. El modelo estructural evalúa el peso y a magnitud de las relaciones entre las distintas variables. Finalmente, para testar las diferencias entre grupos se llevó a cabo un análisis multimuestra para analizar el efecto moderador de las características demográficas de los consumidores.

Antes de proceder al análisis de las hipótesis de investigación, se evaluó la validez del modelo propuesto por Martínez y Rodríguez del Bosque (2013). En primer lugar, la validez convergente del modelo se testó a través de los estadísticos alfa de Cronbach y el análisis de la varianza extraída (AVE). Tal y como se puede observar en la Tabla 2 estos estadísticos superan los valores recomendados de 0.7 y 0.5 respectivamente. Siguiendo a Hair et al. (2010) se utilizó una carga estandarizada de 0.5 para evaluar los ítems de medición. Tal y como puede verse en la Tabla 2 no hubo necesidad de eliminar ningún ítem de las escalas propuestas inicialmente puesto que todas las cargas estandarizadas superaban el valor anterior. La validez discriminante del modelo se evaluó siguiendo el modelo propuesto por Fornell y Larcker (1981). La Tabla 3 muestra adecuados valores de validez discriminante. Para finalizar, los índices de bondad de ajuste del modelo (NFI=Índice de ajuste normado; TLI=Índice de Tucker-Lewis; CFI=Índice comparativo de ajuste; IFI=Índice incremental de ajuste RMSEA= Error cuadrático medio de aproximación) presentan valores muy aceptables $(\mathrm{NFI}=0.916$; $\mathrm{TLI}=0.924$; $\mathrm{CFI}=0.942$; RMSEA=0.07).

Tabla 2

VALIDEZ CONVERGENTE DEL MODELO FINAL

\begin{tabular}{|c|c|c|c|c|c|}
\hline Constructo & Item & $\begin{array}{c}\text { Lambda } \\
\text { estandarizado }\end{array}$ & $\begin{array}{c}\alpha \text { de } \\
\text { Cronbach }\end{array}$ & AVE & \\
\hline Asociaciones de & $\mathrm{RSC} 1$ & 0.650 & & & \\
\hline RSC & $\mathrm{RSC} 2$ & 0.719 & 0.72 & 0.54 & \\
\hline (RSC) & $\mathrm{RSC} 3$ & 0.669 & & & \\
\hline \multirow{4}{*}{$\begin{array}{l}\text { Identificación } \\
\text { C-E } \\
\text { (IDE) }\end{array}$} & IDE1 & 0.764 & \multirow{4}{*}{0.89} & \multirow{4}{*}{0.70} & \\
\hline & IDE2 & 0.816 & & & \\
\hline & IDE3 & 0.894 & & & \\
\hline & IDE4 & 0.908 & & & \\
\hline \multirow{5}{*}{$\begin{array}{c}\text { Confianza } \\
\text { (CON) }\end{array}$} & CON1 & 0.774 & \multirow{5}{*}{0.89} & \multirow{5}{*}{0.64} & $* \mathrm{NFI}=0.916$ \\
\hline & CON2 & 0.854 & & & $\mathrm{TLI}=0.924$ \\
\hline & CON3 & 0.850 & & & $\mathrm{CFI}=0.942$ \\
\hline & CON4 & 0.792 & & & IFI $=0.942$ \\
\hline & CON5 & 0.708 & & & RMSEA $=0.071$ \\
\hline \multirow{4}{*}{$\begin{array}{l}\text { Satisfacción } \\
\text { (SAT) }\end{array}$} & SAT1 & 0.889 & \multirow{4}{*}{0.88} & \multirow{4}{*}{0.70} & \\
\hline & SAT2 & 0.867 & & & \\
\hline & SAT3 & 0.895 & & & \\
\hline & SAT4 & 0.656 & & & \\
\hline \multirow{4}{*}{$\begin{array}{c}\text { Lealtad } \\
\text { (LEA) }\end{array}$} & LEA1 & 0.753 & \multirow{4}{*}{0.88} & \multirow{4}{*}{0.65} & \\
\hline & LEA2 & 0.658 & & & \\
\hline & LEA3 & 0.899 & & & \\
\hline & LEA4 & 0.899 & & & \\
\hline
\end{tabular}

*NFI=Normed fit index; TLI=Tucker-Lewis index; CFI=Comparative fit index; IFI=Incremental fit index; RMSEA= Root mean square error of approximation 
Tabla 3

VALIDEZ DISCRIMINANTE

\begin{tabular}{cccccc}
\hline & RSC & IDE & CON & SAT & LEA \\
\hline RSC & $\mathbf{0 . 7 3}$ & & & & \\
IDE & 0.39 & $\mathbf{0 . 8 3}$ & & & \\
CON & 0.22 & 0.06 & $\mathbf{0 . 8 0}$ & & \\
SAT & 0.35 & 0.27 & 0.50 & $\mathbf{0 . 8 3}$ & \\
LEA & 0.29 & 0.63 & 0.62 & 0.67 & $\mathbf{0 . 8 1}$ \\
\hline
\end{tabular}

La diagonal principal muestra la raíz cuadrada del AVE. El resto de valores corresponde a las correlaciones entre los constructos.

Tabla 4

MODELO ESTRUCTURAL

\begin{tabular}{clc}
\hline Hipótesis & \multicolumn{1}{c}{ Relación estructural } & $\begin{array}{c}\text { Coeficiente } \\
\text { estandarizado (t-valor) }\end{array}$ \\
\hline $\mathrm{H}_{1}$ & Confianza $\rightarrow$ Lealtad & $0.55(9.12)^{*}$ \\
$\mathrm{H}_{2}$ & Identificación C-E $\rightarrow$ Lealtad & $0.41(3.66)^{*}$ \\
$\mathrm{H}_{3}$ & Satisfacción $\rightarrow$ Lealtad & $0.34(2.75)^{*}$ \\
$\mathrm{H}_{4}$ & $\mathrm{RSC} \rightarrow$ Identificación C-E & $0.24(3.74)^{*}$ \\
$\mathrm{H}_{5}$ & $\mathrm{RSC} \rightarrow$ Confianza & $0.45(4.95)^{*}$ \\
$\mathrm{H}_{6}$ & $\mathrm{RSC} \rightarrow$ Satisfacción & $0.67(11.02)^{*}$ \\
$\mathrm{H}_{7}$ & Identificación C-E $\rightarrow$ Satisfacción & $0.37(3.65)^{*}$ \\
$\mathrm{H}_{8}$ & Confianza $\rightarrow$ Identificación C-E & $0.08(1.56)$ \\
$\mathrm{H}_{9}$ & Confianza $\rightarrow$ Satisfacción & $0.67(4.38)^{*}$ \\
\hline $\mathrm{NFI}=0.916$ & $\mathrm{TLI}=0.924 \quad \mathrm{CFI}=0.942 \quad \mathrm{IFI}=0.942$ & $\mathrm{RMSEA}=0.07$ \\
\hline
\end{tabular}

\subsection{Análisis del modelo estructural}

Una vez validado el instrumento de medida, la estimación de las relaciones causales se realizó a través del software estadístico AMOS 23 (Arbuckle, 2014). Los resultados del análisis confirman todas las relaciones propuestas excepto la relación entre la confianza de los consumidores y su nivel de identificación con la empresa $(\beta=0.08, \mathrm{p}<0.05)$. La estimación de los resultados demuestra que la confianza de los consumidores $\left(\beta=0.56^{*}, \mathrm{p}<0.05\right)$, su identificación con la empresa $\left(\beta=0.41^{*}, \mathrm{p}<0.05\right)$ y su satisfacción $\left(\beta=0.33^{*}, \mathrm{p}<0.05\right)$ poseen un efecto positivo y directo sobre su grado de lealtad. Los resultados también demuestran que las asociaciones de RSC poseen una influencia positiva sobre la identificación del consumidor con la empresa $\left(\beta=0.24^{*}, \mathrm{p}<0.05\right)$, su confianza $\left(\beta=0.46^{*}, \mathrm{p}<0.05\right)$ y su satisfacción $\left(\beta=0.68^{*}, \mathrm{p}<0.05\right)$. Finalmente, la identificación del consumidor con la empresa posee un efecto directo sobre la satisfacción $\left(\beta=0.38^{*}, \mathrm{p}<0.05\right)$ y la confianza sobre la satisfacción $\left(\beta=0.67^{*}, p<0.05\right)$. Además, los índices de bondad de ajuste exceden 
en todos los casos el valor recomendado de $0.9\left(\chi^{2}=482.7, \mathrm{p}<0.005 ; \mathrm{df}=162 ; \mathrm{NFI}=0.916\right.$; $\mathrm{IFI}=0.942 ; \mathrm{TLI}=0.924 ; \mathrm{CFI}=0.942 ; \mathrm{RMSEA}=0.07)$. La Tabla 4 muestra estos resultados.

\subsection{Análisis multimuestra}

Tras realizar la estimación del modelo global para todos los individuos se procedió a la comprobación de la existencia de diferencias en función de las características demográficas ya descritas. Así, se llevó a cabo un análisis multimuestra para cada variable moderadora utilizando el programa estadístico AMOS 23. Para ello, se estimó el modelo para cada uno de los diferentes grupos atendiendo a cada una de las variables moderadoras. Así, obtuvimos los parámetros para cada uno de los grupos de forma separada y una media global de ajuste. La principal ventaja de este tipo de estimaciones radica en la estimación simultánea del modelo propuesto en cada grupo y de modelos que incluyen restricciones en las relaciones entre las variables para finalmente saber si los coeficientes estimados varían o no entre grupos (San Martín, 2005).

Como paso previo, se testó la invarianza factorial del modelo propuesto. El objetivo de este análisis previo es comprobar que los constructos son comprendidos de igual modo por cada uno de los grupos de consumidores y que por tanto el modelo es comparable entre dichos grupos. Se comprueba que los valores en el incremento del estadístico $\chi^{2}(p>0.05)$ son no significativos por lo que se confirma la invarianza factorial. A continuación, se analizó la influencia de cada una de las tres variables moderadoras (género, edad y nivel educativo) en el modelo general propuesto. Los modelos sin restricciones ajustan bien y presentan algunas diferencias entre grupos como se comentará a continuación. Posteriormente, se incluyó la restricción de que todos los coeficientes eran iguales. Si comparamos los valores del estadístico $\chi^{2}$ entre los modelos no restringidos y los restringidos los valores resultaron ser significativos (Tabla 5). Estos resultados sugieren que el impacto de las variables moderadoras en el modelo propuesto son significativas. Se analizó también la relación crítica para las diferencias de modo que se estimó el estadístico z para las comparaciones por pares. Los resultados se presentan en la Tabla 6.

De este modo, se observa que el género del consumidor influye en la formación de lealtad basada en aspectos de RSC. Particularmente, el género modera las relaciones entre las asociaciones de RSC y la satisfacción, la satisfacción y la lealtad y la relación entre la identificación C-E y la lealtad. En general, todas las relaciones son más fuertes entre las mujeres. Cuando se considera la variable edad como moderados, los resultados muestran que esta variable demográfica modera tres relaciones en el proceso de formación de lealtad: el efecto de las asociaciones de RSC en la identificación C-E, la influencia de la satisfacción en la lealtad y de la confianza sobre la lealtad. Parece como la relación entre las asociaciones de RSC y el grado de identificación es mayor en los consumidores más jóvenes mientras que el efecto de la satisfacción y la confianza sobre la lealtad es mayor para los consumidores de mayor edad. De igual modo el nivel educativo de los consumidores modera tres relaciones del modelo propuesto: el efecto de la satisfacción y la confianza en la lealtad y la influencia de las asociaciones de RSC en la confianza. En vista de estos resultados ninguna de las hipótesis propuestas puede ser rechazada. 
Tabla 5

ANÁLISIS MULTIMUESTRA Y TEST DE DIFERENCIAS DEL $\chi 2$

\begin{tabular}{clccc}
\hline Grupos & \multicolumn{1}{c}{ Modelos } & $\chi^{\mathbf{2}}$ & df & $\Delta \chi^{\mathbf{2}}(\mathbf{d f})$ \\
\hline \multirow{2}{*}{ Género } & Modelo no restringido & 617.6 & 322 & $42.06(13) ; \mathrm{p}<0.05$ \\
& Modelo restringido & 659.6 & 335 & (significativo) \\
\hline \multirow{2}{*}{ Edad } & Modelo no restringido & 672.3 & 322 & $23.60(13) ; \mathrm{p}<0.05$ \\
& Modelo restringido & 695.9 & 335 & (significativo) \\
\hline \multirow{2}{*}{ Nivel educativo } & Modelo no restringido & 639.7 & 322 & $18.32(13) ; \mathrm{p}<0.05$ \\
& Modelo restringido & 658.0 & 335 & (significativo) \\
\hline
\end{tabular}

Tabla 6

EFECTO MODERADOR DE LAS VARIABLES DEMOGRÁFICAS

\begin{tabular}{|c|c|c|c|}
\hline Relaciones & Coeficientes & estandarizados & Test-z \\
\hline $\begin{array}{l}\text { Género } \\
\text { RSC-Confianza } \\
\text { RSC-Satisfacción } \\
\text { RSC-Identificación } \\
\text { Confianza-Satisfacción } \\
\text { Confianza-Identificación } \\
\text { Identificación-Satisfacción } \\
\text { Satisfacción-Lealtad } \\
\text { Identificación-Lealtad } \\
\text { Confianza-Lealtad }\end{array}$ & $\begin{array}{l}\text { Hombres }(n=190) \\
0.493 \\
0.650 \\
0.253 \\
0.638 \\
0.077 \\
0.380 \\
0.309 \\
0.364 \\
0.026\end{array}$ & $\begin{array}{l}\text { Mujeres }(n=192) \\
0.437 \\
0.719 \\
0.217 \\
0.656 \\
0.005 \\
0.383 \\
0.364 \\
0.496 \\
0.041\end{array}$ & $\begin{array}{l}-0.489 \\
-1.988^{*} \\
0.333 \\
0.085 \\
0.751 \\
-0.572 \\
2.174 * * \\
-1.858^{*} \\
0.295 \\
\end{array}$ \\
\hline $\begin{array}{l}\text { Edad } \\
\text { RSC-Confianza } \\
\text { RSC-Satisfacción } \\
\text { RSC-Identificación } \\
\text { Confianza-Satisfacción } \\
\text { Confianza-Identificación } \\
\text { Identificación-Satisfacción } \\
\text { Satisfacción-Lealtad } \\
\text { Identificación-Lealtad } \\
\text { Confianza-Lealtad }\end{array}$ & $\begin{array}{l}\text { Jóvenes }(n=186) \\
0.443 \\
0.686 \\
0.209 \\
0.623 \\
0.063 \\
0.318 \\
0.356 \\
0.357 \\
0.406\end{array}$ & $\begin{array}{l}\text { Adultos }(n=196) \\
0.482 \\
0.670 \\
0.157 \\
0.684 \\
0.039 \\
0.411 \\
0.491 \\
0.434 \\
0.601\end{array}$ & $\begin{array}{l}-0.968 \\
-0.403 \\
1.692^{*} \\
-0.703 \\
0.592 \\
1.108 \\
1.734^{*} \\
-0.997 \\
1.672^{*} \\
\end{array}$ \\
\hline $\begin{array}{l}\text { Nivel educativo } \\
\text { RSC-Confianza } \\
\text { RSC-Satisfacción } \\
\text { RSC-Identificación } \\
\text { Confianza-Satisfacción } \\
\text { Confianza-Identificación } \\
\text { Identificación - Satisfacción } \\
\text { Satisfacción-Lealtad } \\
\text { Identificación-Lealtad } \\
\text { Confianza-Lealtad }\end{array}$ & $\begin{array}{l}\text { Bajo }(n=232) \\
0.481 \\
0.683 \\
0.173 \\
0.680 \\
0.085 \\
0.361 \\
0.395 \\
0.417 \\
0.647\end{array}$ & $\begin{array}{l}\text { Alto }(\mathrm{n}=150) \\
0.450 \\
0.632 \\
0.263 \\
0.691 \\
0.063 \\
0.372 \\
0.302 \\
0.456 \\
0.502\end{array}$ & $\begin{array}{l}-1.690^{*} \\
-0.843 \\
-0.306 \\
0.226 \\
-0.680 \\
1.279 \\
-2.041^{* *} \\
1.041 \\
1.523^{*}\end{array}$ \\
\hline
\end{tabular}




\section{CONCLUSIONES, IMPLICACIONES PARA LA GESTIÓN Y FUTURAS LÍNEAS DE INVESTIGACIÓN}

Los resultados de esta investigación apoyan la propuesta de que las características demográficas de los consumidores moderan su proceso de formación de la lealtad basada en aspectos de RSC. En concreto, las tres características demográficas que se analizaron moderan el proceso. Los resultados demuestran que el género, edad y nivel educativo de los consumidores influyen en la forma en que este grupo forma su lealtad hacia los hoteles teniendo en cuenta aspectos socialmente responsables.

En primer lugar, los resultados muestran como las mujeres tienden a estar más satisfechas que los hombres debido al desarrollo de iniciativas de RSC por parte de las empresas. Es decir, son las mujeres las que desarrollan unos niveles de satisfacción más elevados por el mero hecho de que las compañías desarrollen este tipo de iniciativas. Teniendo en cuenta que las iniciativas socialmente responsables buscan favorecer la sociedad, proteger el medioambiente e incrementar la calidad de vida de distintos colectivos, estos resultados coinciden con investigaciones previas que demuestran que las mujeres se preocupan más por el bienestar de los demás poniendo un mayor énfasis en las relaciones interpersonales (Konrad et al., 2000). Igualmente, se demuestra como en este segmento la relación entre su grado de identificación y su grado de lealtad es más fuerte que en el caso de los hombres. Lo mismo sucede en la relación entre su nivel de satisfacción y su nivel de confianza. Estos resultados apoyan los postulados de la Teoría del Rol Social reconociendo que las mujeres aportan una mayor relevancia a las relaciones personales al tiempo que son menos analíticas a la hora de evaluar la información que les llega de las organizaciones (McColl-Kennedy et al., 2003). Por ello, en el segmento de consumidores femeninos la lealtad basada en aspectos de RSC se basará más en su experiencia con el servicio que en las propias comunicaciones de la compañía.

En segundo lugar, los resultados muestran como el segmento de consumidores más jóvenes tiende a identificarse en mayor medida con las empresas hoteleras que desarrollan iniciativas socialmente responsables. Siguiendo los postulados de la Teoría del Procesamiento de la Información este resultado es lógico si tenemos en cuenta que las generaciones más jóvenes son las más receptivas a la información de carácter social y ético, lo que provoca que estos aspectos incidan en mayor medida en el nivel de identificación con la compañía de lo que les ocurre a los consumidores de mayor edad (Patterson, 2007). Igualmente, se demuestra como en el segmento de los consumidores más mayores la relación entre su grado de satisfacción y confianza y su nivel de lealtad hacia la compañía son más fuertes que en el segmento de consumidores más jóvenes. Estos resultados apoyan investigaciones previas que demuestran que a la hora de tomar decisiones, las personas mayores confían más en su propia experiencia y satisfacción con el servicio prestado (Homburg y Giering, 2001). Los jóvenes en cambio tienden a prestar mayor atención a la información ofrecida por la empresa. En este mismo sentido, Walsh et al. (2008), siguiendo de nuevo los postulados de la Teoría del Procesamiento de la Información, proponen que cabe esperar que los consumidores de mayor edad se apoyen en menos criterios que los jóvenes a la hora de desarrollar su lealtad. Esto 
demuestra que la satisfacción y la confianza adquiridas con experiencias previas serán factores determinantes en el sector más maduro de consumidores y no así en el sector más joven puesto que estos últimos tenderán a buscar más información que pueda influirles en su grado de lealtad.

Respecto al nivel educativo de los consumidores, los resultados demuestran que la relación entre las asociaciones de RSC y su nivel de confianza es más fuerte para los consumidores con un nivel educativo inferior. Lo mismo sucede con la relación entre su grado de confianza y su grado de satisfacción y lealtad. Estos resultados son consistentes con los postulados de la Teoría del Procesamiento de la Información en el sentido de que los consumidores con un menor nivel educativo consideran un menor rango de señales informativas cuando evalúan un producto o compañía (Walsh y Mitchell, 2008). En este sentido, este grupo de consumidores se preocupa en mayor medida por señales no relacionadas directamente con características funcionales de los productos en comparación con el grupo de consumidores con mayor nivel educativo quienes si aportan una mayor relevancia a los aspectos funcionales de los productos/servicios.

Este estudio presenta varias implicaciones prácticas para los gestores de establecimientos hoteleros. Los resultados sugieren que con el fin de mejorar la fidelidad de los clientes basados en la RSC en la industria hotelera, las comunicaciones sobre este tipo de aspectos deben adaptarse a los diferentes grupos de consumidores y comunicar las iniciativas que llevan a cabo de maneras diferenciadas para cada segmento del mercado. En este sentido, y en vista de los resultados obtenidos, resultaría interesante que las campañas de comunicación se dirigieran principalmente a los consumidores de género masculino y de menor edad. Esto se conseguiría fácilmente utilizando canales de comunicación personales (e.g, mailing, redes sociales...) en lugar de recurrir a medios de comunicación masivos. Igualmente, las campañas de comunicación basadas en aspectos sociales parece que tendrían un mayor impacto en los consumidores con un menor nivel educativo puesto que los consumidores con mayor nivel de estudios se basan en aspectos más funcionales como precio y calidad. De este modo, los gerentes deberían desarrollar planes para identificar grupos de clientes con características personales similares y comparar sus intenciones hacia la empresa basados en aspectos de RSC.

Así mismo, y en vista de los resultados obtenidos se recomienda sensibilizar a los segmentos más reacios a este tipo de iniciativas. Por ello los gestores podrían "educar" a todos los clientes para que comprendan las verdaderas motivaciones tras las iniciativas socialmente responsables desarrolladas por las compañías del sector y los beneficios que de ellas se derivan, no sólo para las empresas sino para las comunidades locales y el medioambiente. De este modo, se recomienda realizar campañas informativas y/o seminarios así como aportar información en los propios establecimientos hoteleros bien a través de folletos o medios impresos o a través de los propios empleados.

No obstante, este estudio presenta algunas limitaciones que también sugieren nuevos trabajos de investigación. En primer lugar, el contexto de este estudio fue el sector turístico, en concreto las empresas hoteleras. Al elegir un área de investigación concreta este estudio permite detectar efectos moderadores en un contexto específico pero investigaciones futuras podrían también explorar otras industrias, sobre todo por el 
marcado contextual que se ha reconocido en la práctica de la RSC. Igualmente, estudios futuros podrían considerar la inclusión de variables moderadoras adicionales que no se han tenido en cuenta en el presente estudio. Por ejemplo, sería interesante incluir en el análisis los rasgos psicológicos de los consumidores para explicar mejor el proceso de formación de la lealtad. Así mismo, estudios futuros podrían integrar características propias de las empresas (p.e., imagen o reputación), ya que estos activos intangibles pueden afectar el proceso de formación de la lealtad.

\section{BIBLIOGRAFÍA}

ANDERSON, J.C. y GERBING, D.W. (1988). «Structural equation modelling in practice: A review and recommended two-step approach», Psychological Bulletin, vol. $103, n^{\circ} 3$, pp. 411-423.

ARBUCKLE, J.L. (2014). IBM®SPSS® AMOSTM 23 User's Guide. IBM

ARCHER, J. (1996). «Sex differences in social behavior: Are the social role and evolutionary explanations compatible? », American Psychologist, vol. 51, n 9, pp. 909-917.

BALDERJAHN, I. (1988). «Personality variables and environmental attitudes as predictors of ecologically responsible consumption patterns», Journal of Business Research, vol. 17, $\mathrm{n}^{\mathrm{o}} 1$, pp. 51-56.

BARON, R.M. y KENNY, D.A. (1986). «Moderator-mediator variables distinction in social psychological research: Conceptual, strategic, and statistical considerations», Journal of Personality and Social Psychology, vol. 51, nº 6, pp. 1173-1182.

BOHDANOWICZ, P. y ZIENTARA, P. (2009). «Hotel companies' contribution to improving the quality of life of local communities and the well-being of their employees», Tourism and Hospitality Research, vol. 9, n 2, pp. 147-158.

BROWN, T.J. y DACIN, P.A. (1997). «The company and the product: corporate association and consumer product responses», Journal of Marketing, vol. 61, pp. 68-84.

BUSS, D. M. (1996). The evolutionary psychology of human social strategies. Higgins, Edward Tory (Ed); Kruglanski, Arie W. (Ed). Social psychology: Handbook of basic principles. New York, NY, US: Guilford Press, pp. 3-38.

CHAN, E.S.W. (2011). «Implementing environmental management systems in small and medium-sized hotels: Obstacles», Journal of Hospitality \& Tourism Research, vol. 35, no 1, pp. 3-23.

CRONIN, J.J., BRADY, M.K. y HULT, G.T.M. (2000). «Assessing the effects of quality, value, and customer satisfaction on consumer behavioral intentions in service environments», Journal of Retailing, vol. 76, n 2, pp. 193-218.

DIAMANTOPOULOS, A., SCHLEGELMILCH, B. B., SINKOVICS, R.R, y BOHLEN, G.M. (2003). «Can socio-demographics still play a role in profiling green consumers? A review of the evidence and an empirical investigation», Journal of Business Research, vol. 56, $\mathrm{n}^{\circ}$ 6, 465-480.

EAGLY, A.H. (1987). Sex differences in social behavior: A social-role interpretation. Hillsdale: Lawrence Erlbaum Associates. 
EVANSCHITZKY, H. y WUNDERLICH, M. (2006). «An examination of moderator effects in the four-stage loyalty model», Journal of Service Research, vol. 8, $\mathrm{n}^{\circ} 4$, pp. 305-345.

FORNELL, C. y LARCKER, D.F. (1981). «Evaluating structural equation models with unobservable variables and measurement error», Journal of Marketing Research, vol. $18, \mathrm{n}^{\circ} 1$, pp. 39-50.

GILLY, M.C. y ZEITHAML, V.A. (1985). «The elderly consumer and adoption of technologies», Journal of Consumer Research, vol. 12, nº 3, pp. 353-357.

HAIR, J.F., BLACK, W.C., BABIN, B.J. y ANDERSON, R.E. (2010). Multivariate Data Analysis. Pearson Prentice-Hall, Upper Saddle River.

HAN, H., HSUB, L.T., LEE, J.S. y SHEUD, C. (2009). «Are lodging customers ready to go green? An examination of attitudes, demographics, and eco-friendly intentions», International Journal of Hospitality Management, vol. 30, $\mathrm{n}^{\mathrm{o}}$ 2, pp. 345-355.

HOLLOWAY, C.J. (2004). Marketing for Tourism. Prentice Hall, Harlow.

HOMBURG, C. y GIERING, A. (2001). «Personal characteristics as moderators of the relationship between Customer satisfaction and loyalty: An empirical analysis», Psychology \& Marketing, vol. 18, $\mathrm{n}^{\circ}$ 1, pp. 43-66.

HENRIQUE, J.L. y DE MATOS, C.A. (2015). «The influence of personal values and demographics variables on customer loyalty in the banking industry», International Journal of Bank Marketing, vol. 33, n 4, pp. 571-587.

KELLEY, S.W., DONNELY, JR., J.H. y SKINNER, S.J. (1990). «Customer participation in service production and delivery», Journal of Retailing, vol. 66, $\mathrm{n}^{\circ} 3$, pp. 315-335.

KONRAD, A.M., RITCHIE JR., J.E., LIEB, P. y CORRIGALL, E. (2000). «Sex differences and similarities in job attribute preferences: a meta-analysis». Psychological Bulletin, vol. 126, $\mathrm{n}^{\mathrm{O}} 4$, pp. 593-641.

LAROCHE, M., BERGERON, J. y BARBARO-FORLEO, G. (2001). «Targeting consumers who are willing to pay more for environmentally friendly products», Journal of Consumer Marketing, vol. 18, $\mathrm{n}^{\circ}$ 6, pp. 503-520.

LUIZ J.H. y DE MATOS, C.A. (2015) «The influence of personal values and demographic variables on customer loyalty in the banking industry», International Journal of Bank Marketing, vol. 33, $\mathrm{n}^{\circ}$ 4, pp.571-587.

MAEL, F. y ASHFORTH. B. (1992). «Alumni and their alma mater: A partial test of the reformulated model of organizational identification», Journal of Organizational Behavior, vol. 13, n 2, pp. 103-123.

MARTÍNEZ, P., PÉREZ, A. y RODRÍGUEZ DEL BOSQUE, I. (2012). «Análisis de la práctica corporativa de la responsabilidad social en el sector turístico: Un estudio de casos», Cuadernos de Turismo, no ${ }^{\circ} 30$, pp. 145-164.

MARTÍNEZ, P. y RODRÍGUEZ DEL BOSQUE, I. (2013). «CSR and customer loyalty: The roles of trust, customer identification with the company and satisfaction», International Journal of Hospitality Management, vol. 35, pp. 89-99.

MITTAL, V. y KAMAKURA, W. A. (2001). «Satisfaction, repurchase intent, and repurchase behavior: Investigating the moderating effect of customer characteristics», Journal of Marketing Research, vol. 38, n 1, pp. 131-142. 
MORGAN, R.M. y HUNT, S.D. (1994). «The commitment-trust theory of relationship marketing», Journal of Marketing, vol. 58, pp. 20-38.

PATTERSON, P. (2007), «Demographic correlates of loyalty in a service context», Journal of Services Marketing, vol. 21, n 2, pp. 112-121.

PÉREZ, A. y RODRÍGUEZ DEL BOSQUE, I. (2013). «Customer personal features as determinants of the formation process of corporate social responsibility perceptions», Psychology \& Marketing, vol. 30, nº 10, pp. 903-917.

PÉREZ, A. y RODRÍGUEZ DEL BOSQUE, I. (2015). «How customers construct corporate social responsibility images: Testing the moderating role of demographic characteristics», Business Research Quarterly, vol. 18, n 2, pp. 127-141.

PIVATO, S., MISANI, N. y TENCATI, A. (2008). «The impact of corporate social responsibility on consumer trust: the case of organic food», Business Ethics: A European Review, vol. 17, nº 1, pp. 3-12.

QUAZI, A.M. (2003). «Identifying the determinants of corporate managers perceived social obligations», Management Decision, vol. 41, $\mathrm{n}^{\circ}$ 9, 822-831.

ROBERTS, J.A. (1996). «Green consumers in the 1990s: profile and implications for advertising», Journal of Business Research, vol. 36, n 3, pp. 217-231.

SAAD, G. y GILL, T. (2000). «Applications of evolutionary psychology in marketing», Psychology \& Marketing, vol. 17, $\mathrm{n}^{\circ}$ 12, pp. 1005-1034.

SANDAHL, D.M. y ROBERTSON, R. (1989). «Social determinants of environmental concern: specification and test of the model», Environment and Behavior, vol. 21, $\mathrm{n}^{\mathrm{o}} 1$, pp. 57-81.

SAN MARTÍN, S. (2006). «La generación de la confianza del consumidor en el establecimiento comercial. Una perspectiva multinivel», Revista Europea de Dirección y Economía de la Empresa, vol. 15, n 1, pp. 201-224.

SIRDESHMUKH, D., JAPDIG, S. y BERRY, S. (2002). «Customer trust, value, and loyalty in relational exchanges», Journal of Marketing, vol. 66, $\mathrm{n}^{\circ}$ 1, pp. 15-37.

SO, K.K.F, KING, C., SPARKS, B. y WANG, Y. (2013). «The influence of customer brand identification on hotel brand evaluation and loyalty development», International Journal of Hospitality Management, vol. 34, pp. 31-41.

VÁZQUEZ-CARRASCO, R. y FOXALL, G.R. (2006). «Influence of personality traits on satisfaction, perception of relational benefits, and loyalty in a personal service context», Journal of Retailing and Consumer Services, vol. 13, nº 3, pp. 205-219.

WAKEFIELD, L. y BAKER, J. (1998). «Excitement at the mall: determinants and effects on shopping response», Journal of Retailing, vol. 74, $\mathrm{n}^{\circ}$ 4, pp. 515-39.

WALSH, G., EVANSCHITZKY, H. y WUNDERLICH, M. (2008). «Identification and analysis of moderator variables. Investigating the customer satisfaction-loyalty link», European Journal of Marketing, vol. 42, n 9-10, pp. 977-1.004.

ZEITHAML, V.A., BERRY, L.L. y PARASURAMAN, A. (1996). «The behavioral consequences of service quality», Journal of Marketing, vol. 60, pp. 31-46. 


\section{APÉNDICE}

\begin{tabular}{|c|c|}
\hline Identificador & Items \\
\hline \multicolumn{2}{|c|}{ Asociaciones de RSC } \\
\hline RSC1 & Esta compañía protege el medioambiente \\
\hline $\mathrm{RSC} 2$ & $\begin{array}{l}\text { Esta compañía muestra su compromiso con la sociedad mejorando la cali- } \\
\text { dad de vida de las comunidades en las que opera }\end{array}$ \\
\hline RSC3 & $\begin{array}{l}\text { Esta compañía destina parte de su presupuesto a donaciones a diversas } \\
\text { causas sociales }\end{array}$ \\
\hline \multicolumn{2}{|c|}{ Identificador C-E } \\
\hline IDE1 & $\begin{array}{l}\text { Cuando alguien critica a esta compañía hotelera, siento como si me in- } \\
\text { sultaran }\end{array}$ \\
\hline IDE2 & $\begin{array}{l}\text { Estoy muy interesado en lo que otras personas piensan de esta compañía } \\
\text { hotelera }\end{array}$ \\
\hline IDE3 & Cuando hablo de esta compañía hotelera, digo "nosotros" en lugar de "yo" \\
\hline IDE4 & $\begin{array}{l}\text { Cuando alguien hace un cumplido a esta compañía hotelera, lo tomo } \\
\text { como un cumplido para mí mismo. }\end{array}$ \\
\hline \multicolumn{2}{|l|}{ Satisfacción } \\
\hline SAT1 & Hospedarse en un hotel de esta compañía es genial \\
\hline SAT2 & Mi decisión de hospedarme en esta compañía hotelera fue acertada \\
\hline SAT4 & Creo que hice lo correcto al elegir hospedarme en este hotel \\
\hline SAT4 & Esta compañía hotelera ofrece lo que realmente necesito \\
\hline \multicolumn{2}{|l|}{ Confianza } \\
\hline CON1 & $\begin{array}{l}\text { Los servicios de esta compañía hotelera me generan un sentimiento de } \\
\text { seguridad }\end{array}$ \\
\hline COM2 & Confío en la calidad de esta compañía \\
\hline CON3 & $\begin{array}{l}\text { La contratación de los servicios de esta compañía hotelera es una garantía } \\
\text { de calidad }\end{array}$ \\
\hline CON4 & Esta empresa hotelera está interesada en sus clientes \\
\hline CON5 & Esta compañía hotelera es honesta con sus clientes \\
\hline \multicolumn{2}{|l|}{ Lealtad } \\
\hline LEA1 & $\begin{array}{l}\text { Suelo elegir esta compañía hotelera como primera opción en comparación } \\
\text { con otras compañías del sector }\end{array}$ \\
\hline LEA2 & $\begin{array}{l}\text { Sería costoso en términos de dinero, tiempo y esfuerzo poner fin a la rela- } \\
\text { ción con esta compañía hotelera. }\end{array}$ \\
\hline LEA3 & $\begin{array}{l}\text { Voy a seguir considerando esta compañía hotelera como mi principal op- } \\
\text { ción en un futuro. }\end{array}$ \\
\hline LEA4 & Recomendaría esta compañía hotelera si alguien pidiera mi consejo. \\
\hline
\end{tabular}


